INPLASY

PROTOCOL

To cite: Yang. Was

Antiphospholipid Syndrome A

Risk Factor of Stroke? A

Systemic Review and Meta-

analysis of Cohort Studies

Published in the 21st Century.

Inplasy protocol 202180074.

doi:

10.37766/inplasy2021.8.0074

Received: 19 August 2021

Published: 19 August 2021

Corresponding author:

Mingfei Yang

15544980059@163.com

Author Affiliation:

Department of Neurosurgery, Qinghai Provincial People's Hospital, Xining, Qinghai, 810007, P.R. China.

Support: Department of Qinghai Province.

Review Stage at time of this submission: Completed.

\section{Was Antiphospholipid Syndrome A Risk Factor of Stroke? A Systemic Review and Meta-analysis of Cohort Studies Published in the 21st Century}

Yang, MF1.

Review question / Objective: We intended to perform a systemic review and meta-analysis to verify the hypothesis that APS might increase the occurrence of stroke.

Condition being studied: Studies were identified after literature search of PubMed, Embase and Cochrane. QNewcastle-Ottawa Quality Assessment Scale Cohort Studies. (NOQAS-C) was used to perform quality assessment of studies. The pooled effect with its $95 \%$ confidence interval $(95 \% \mathrm{Cl})$ were calculated by random effects model. I-square (I2) was used to test heterogeneity. Funnel plot was used to evaluate publication bias.

Information sources: Literature search was performed in three public electronic databases of PubMed, Embase and Cochrane.

INPLASY registration number: This protocol was registered with the International Platform of Registered Systematic Review and Meta-Analysis Protocols (INPLASY) on 19 August 2021 and was last updated on 19 August 2021 (registration number INPLASY202180074).

Conflicts of interest:

None declared.

\section{INTRODUCTION}

Review question / Objective: We intended to perform a systemic review and metaanalysis to verify the hypothesis that APS might increase the occurrence of stroke.
Condition being studied: Studies were identified after literature search of PubMed, Embase and Cochrane. QNewcastleOttawa Quality Assessment Scale Cohort Studies.(NOQAS-C) was used to perform 
quality assessment of studies. The pooled effect with its $95 \%$ confidence interval $(95 \% \mathrm{Cl})$ were calculated by random effects model. I-square (12) was used to test heterogeneity. Funnel plot was used to evaluate publication bias.

\section{METHODS}

Participant or population: Participation in exposure group were suffered from APS; Participation in non-exposure group only differed in no APS suffered.

Intervention: Participation in exposure group were suffered from APS.

Comparator: Participation in non-exposure group only differed in no APS suffered.

Study designs to be included: Cohort studies.

Eligibility criteria: Inclusion criteria: (1) Language, regions, publication years of articles were not restricted; (2) Cohort studies; (3) Participation in exposure group were suffered from APS; (4) Participation in non-exposure group only differed in no APS suffered; (5) Endpoint of observation was stroke; (6) Analysis of cohort studies' outcomes were performed completely. Exclusion criteria: (1) Duplication; (2) Reviews, comments, letters, case reports, protocols of clinic trials and conference papers; (3) Animal experiments; (4) Contents of articles were irrelevant to this meta-analysis.

Information sources: Literature search was performed in three public electronic databases of PubMed, Embase and Cochrane.

Main outcome(s): All the original data used to assess the outcomes of cohort study were extracted including hazard ratio(HR), risk ratio(RR), odds ratio(OR) and so on.

Strategy of data synthesis: Meta-analysis was performed using corresponding modules in Software for Statistics and Data Science (Stata, version 15.1; College Station, Texas 77845 USA). The pooled effect with its $95 \% \mathrm{Cl}$ were calculated by random effects model.

Subgroup analysis: To reduce heterogeneity, recalculating the pooled effect of the remaining studies after omitting the study with the lowest quality or perform subgroups analysis.

Sensitivity analysis: Sensitivity analysis was performed to evaluate the stability of overall results by recalculating the pooled effect of the remaining studies after omitting the study with the highest quality or the random effects model was switched to fixed effects model.

Country(ies) involved: People's Republic of China.

Keywords: Antiphospholipid Syndrome, Stroke, Cohort Study, Meta-analysis.

Contributions of each author:

Author 1 - Mingfei Yang. 